\title{
catalysts
}

ISSN 2073-4344

www.mdpi.com/journal/catalysts

Article

\section{Cyclopropanation of 5-(1-Bromo-2-phenyl-vinyl)-3-methyl-4- nitro-isoxazoles under Phase Transfer Catalysis (PTC) Conditions}

\author{
Linda Piras ${ }^{1}$, Maria Moccia ${ }^{1}$, Mauro Cortigiani ${ }^{2}$ and Mauro F. A. Adamo ${ }^{2, *}$
}

1 Consiglio Nazionale delle Ricerche (CNR)-Bari Institute of Crystallography, 70126, Italy;

E-Mails: linda.piras@ic.cnr.it (L.P.); maria.moccia@ic.cnr.it (M.M.)

2 Centre for Synthesis and Chemical Biology (CSCB), Royal College of Surgeons in Ireland (RCSI), 123 St Stephen's Green, Dublin 2, Dublin, Ireland; E-Mail: maurocortigiani@rcsi.ie

* Author to whom correspondence should be addressed; E-Mail: madamo@rcsi.ie;

Tel.: +353-1-402 (ext. 2208); Fax: +353-1-402 (ext. 2168).

Academic Editor: Dario Landini

Received: 27 February 2015 / Accepted: 3 April 2015 / Published: 13 April 2015

\begin{abstract}
Heavily substituted cyclopropane esters were prepared in high yields, complete diastereoselection and average (up to 58\%) enantioselectivity. The reaction described herein entailed reacting 4-nitro-5-bromostyrylisoxazoles, a class of powerful Michael acceptors with malonate esters under the catalysis of $5 \mathrm{~mol} \%$ of a chincona derived phase-transfer catalyst.
\end{abstract}

Keywords: phase transfer catalysis; styrylisoxazoles; Michael initiated ring closing reactions

\section{Introduction}

3-Methyl-4-Nitro-5-styrylisoxazoles 1 [1-9] and 2 [3] (Scheme 1) represent two types of polyfunctional scaffold which hold excellent potential for the generation of diversity (Figure 1) [1-9]. 


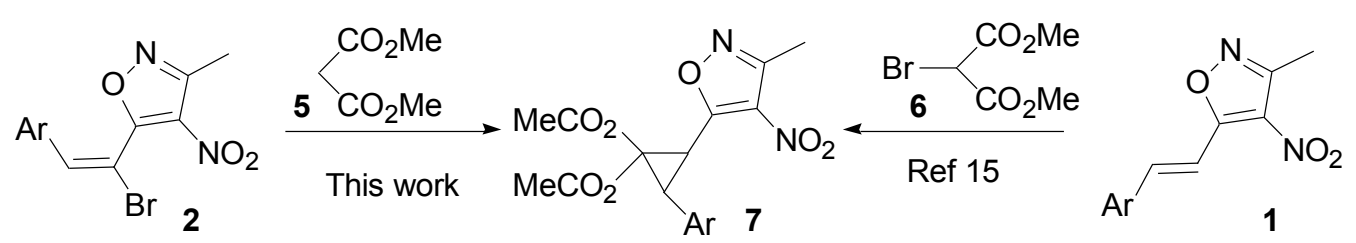

Scheme 1. Preparation of cyclcopropanes 7 via Michael-initiated ring closure (MIRC) processes of 1 and 2.

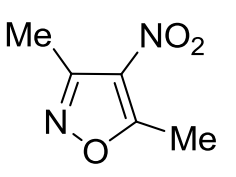

3<smiles>O=C[AlH2]</smiles>

4

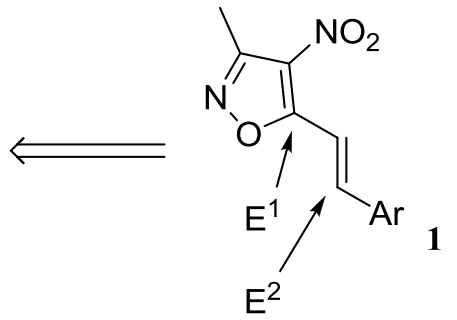

$\mathrm{E}^{2}$

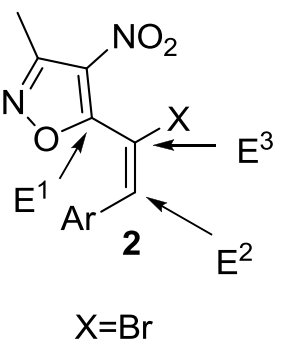

$\mathrm{X}=\mathrm{Br}$

Figure 1. Polyfunctional scaffolds 1 and 2.

Compounds 1 and 2 can be readily prepared from commercially available 3,5-dimethyl-4-nitroisoxazole 3 and aromatic aldehydes 4 (Figure 1) [10]. It has been shown that compounds 1 and 2 can be considered as cinnamate equivalents where the 4-nitro-isoxazole core can be hydrolyzed to give a carboxylate [11].

It is also noteworthy that compounds $\mathbf{1}$ and $\mathbf{2}$ have enhanced reactivity compared to cinnamates due to conjugation of the nitro group at position $\mathrm{C}-4$ of the isoxazole core. Furthermore, compounds $\mathbf{1}$ have two electrophilic centers that can be selectively reacted. Enolates, which are stabilized soft nucleophiles, react at the soft electrophilic center $\mathrm{E}_{2}$, whereas hard nucleophiles such as hydroxide react exclusively at the hard electrophilic center E1 (Figure 1) [1-9,12-14].

Interestingly, isoxazoles 2 (Figure 1), in which a halide is introduced on the exocyclic alkene, hold an additional electrophilic center $\mathrm{E}_{3}$ that increases the number of their possible synthetic applications [3].

For instance, the alkenyl halide moiety in scaffold $\mathbf{2}$ could be employed in various transition metal-mediated $\mathrm{C}-\mathrm{C}$ bond forming reactions including Heck, Sonogashira and related reactions to access dienes or enynes. Cyclopropanes are found in many biologically active molecules $[15,16]$ and hold a unique combination of reactivity and structural properties. It is not surprising, therefore, that stereoselective cyclopropanation has been intensively investigated and enantioselective procedures for the Simmons-Smith reaction and other organometallic-based catalyses reported [17]. Pioneered by Aggarwal and Dai, [18-22] the affirmation of organocatalysis [23,24] delivered a number of high enantioselective cyclopropanation protocols, for example those reported by Gaunt [25-29] and MacMillan [30]. Recently, Michael Initiated Ring Closing (MIRC) processes appeared in the literature [31-40], particularly by W. Wang, [33] Cordova, [34] Y. Wang, [38] Yan, [39] and Tiecco. [40] These methodologies gave densely substituted cyclopropanes in good yields and good to excellent diastereo- and enantio- selectivity. Cyclopropanation has been scarcely studied under phase transfer catalysis [41] with Shiori's [42] and our group [43] providing the only two known examples of high enantioselective procedures. Cyclopropanation reactions which involves a conjugate addition to an electrophilic alkene to produce an enolate, which then subsequently undergoes an intramolecular ring 
closure, are defined as Michael-initiated ring closure (MIRC) reactions. Two types of substrates/reactants can give rise to MIRC reactions.

The first type involves formation of cyclopropanes such as 7 (Scheme 1) via Michael addition of a nucleophile containing a leaving group to an activated alkene. For example, our group has recently reported a highly enantioselective cyclopropanation of 3-methyl-4-nitro-5-styrylisoxazoles 1 that reacted with bromomalonate 6 under the catalysis of Cinchona based phase transfer catalysts [43]. The second type of MIRC processes involves the formation of cyclopropanes by nucleophilic addition to electrophilic substrates containing a leaving group, for example a bromide as in compound $\mathbf{2}$. Herein we report the results of our studies on the reaction of bromostyreneisoxazoles $\mathbf{2}$ and malonate $\mathbf{5}$ under the catalysis of Cinchona based phase transfer catalysts.

\section{Results and Discussion}

We first investigated the addition of dimethyl malonate $5 \mathbf{a}$ to $2 \mathbf{a}$ in the presence of $\mathrm{K}_{3} \mathrm{PO}_{4} 50 \% \mathrm{w} / \mathrm{w}$ as inorganic base, toluene as organic solvent and quaternary ammonium salts derived from Cinchona alkaloids as catalysts (Table 1). The choice of toluene and phosphate arose from a preliminary screening which identified these as the most suitable condition to obtain desired cyclopropane 7 in high yields.

These experiments afforded cyclopropane $7 \mathbf{a}$ with in high conversion even with only 0.05 equiv. of catalyst loading, but with enantioselectivity up to a maximum of $42 \%$. Importantly, cyclopropane $7 \mathbf{a}$ was always obtained as a single diastereoisomer. The higher enantiomeric excess was obtained with catalyst $N$-benzylquininium chloride (Table 1 , entry 1 ). The second generation catalyst $O$-Allyl- $N-9$ anthracenylmethylcinchonidinium bromide, provided high yields of desired $\mathbf{7 a}$, but in an almost racemic form (Table 2, entry 5). The reason for this may lay in the peculiar mode of action of these catalytic species (see Figure 2) in which a free $\mathrm{OH}$ is required to provide a key H-bond with the enolate. [43] Based on the results collected on $\mathrm{N}$-benzylquininium catalyst series, a series of $\mathrm{N}$ benzylquininium salts was prepared containing various functional groups at the benzyl ortho-position and employed as catalysts to promote the Michael addition. These catalysts provided cyclopropane 7a in similar enantiopurity as commercially available $N$-benzylquininium chloride (Table 1, entries 6-9).

In order to increase the enantioselectivity of this reaction, compound $\mathbf{2 a}$ was reacted with malonates bearing alkyl groups of increasing steric hindrance (Table 2); diethyl malonate $\mathbf{5 b}$ furnished cyclopropane 9 in $40 \%$ ee (Table 2, entry 2); the use of bulkier nucleophiles, such as diisopropyl malonate, dibenzyl malonate and diphenyl malonate provided compounds $\mathbf{1 1 - 1 3}$ in lower enantioselectivity (Table 2, entries 3-5).

Table 1. Representative results from the screening of Cinchona derived catalysts

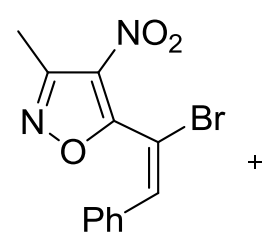

$2 a$

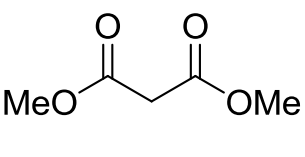

$5 a$
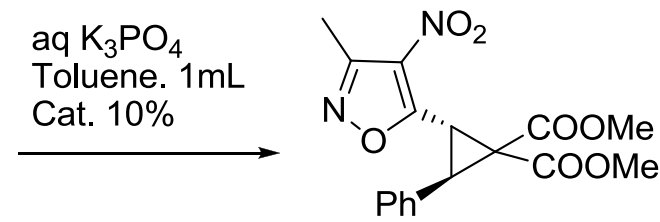

$7 a$ 
Table 1. Cont.

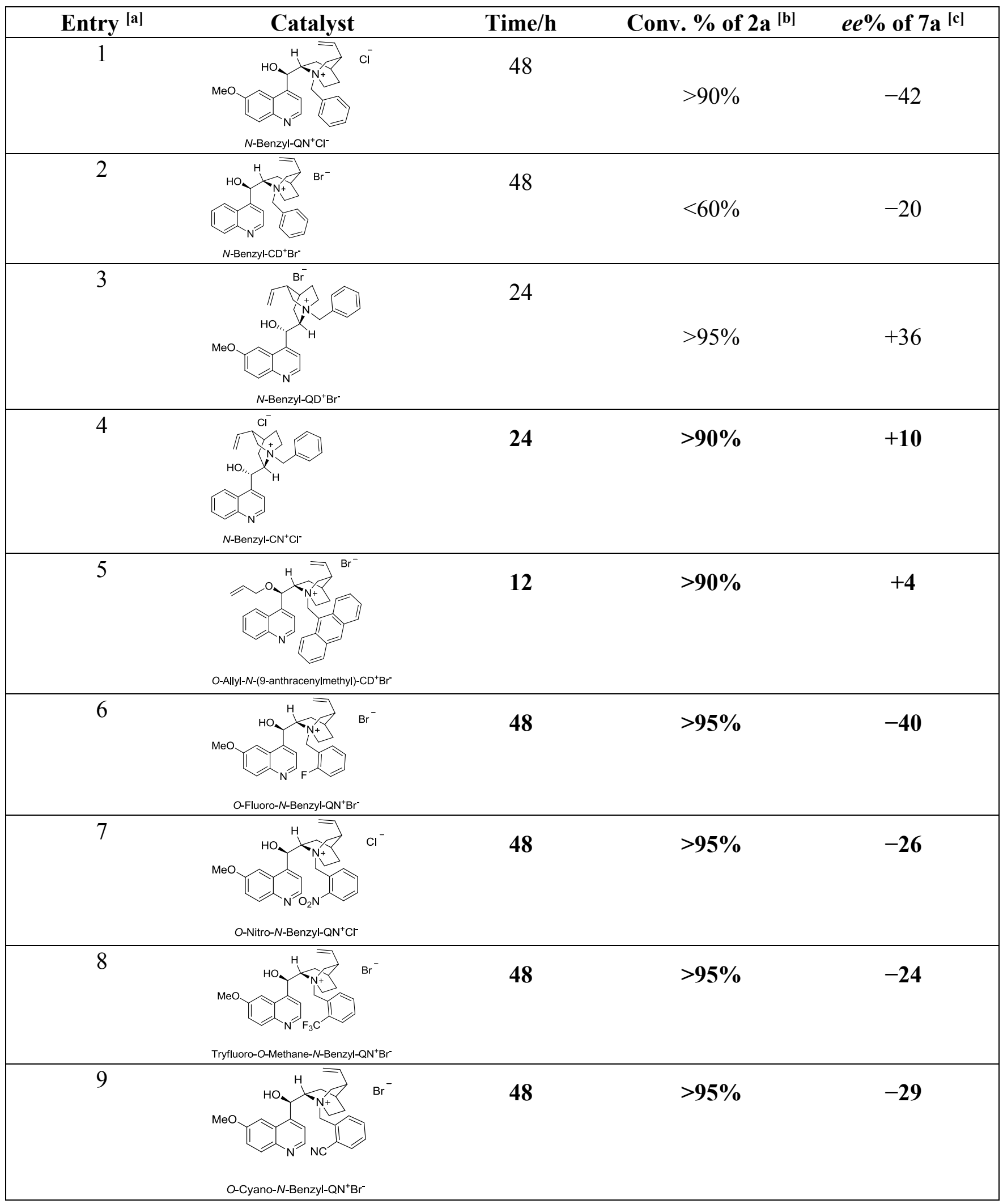

[a] Conditions: Bromostyrylisoxazole 2a $(0.1 \mathrm{mmol})$, malonate 5a $(0.2 \mathrm{mmol})$, cat. $(0.005 \mathrm{mmol}), \mathrm{K}_{3} \mathrm{PO}_{4}$ $50 \% w / w(1 \mathrm{mmol})$, toluene $(1 \mathrm{~mL})$, r.t.. [b] Conversions were determined by NMR analysis. [c] The enantiomeric excess (ee) of the products was determined by chiral stationary phase HPLC. 
Table 2. Catalytic (MIRC) reaction of 2a with dialkyl malonates ${ }^{[a]}$.

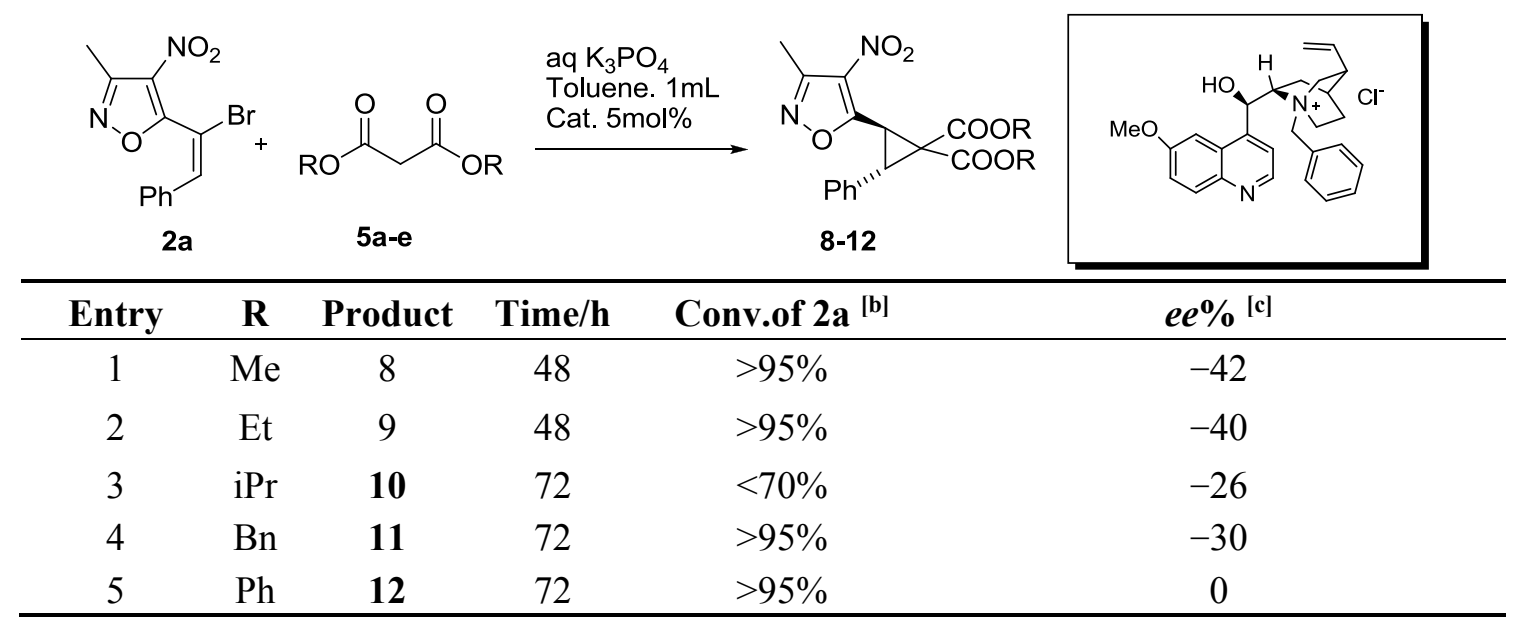

[a] Conditions: Bromostirylisoxazole 2a $(0.1 \mathrm{mmol})$, malonate $\mathbf{5 a}-\mathbf{e}(0.2 \mathrm{mmol})$, cat. $(0.005 \mathrm{mmol}), \mathrm{K}_{3} \mathrm{PO}_{4}$ $50 \% w / w(1 \mathrm{mmol})$, toluene $(1 \mathrm{~mL})$, r.t. [b] Conversions were determined by NMR analysis. [c] The enantiomeric excess $(e e)$ of the products was determined by chiral stationary phase HPLC.

The scope of reaction was shown by reacting styryilisoxazoles $\mathbf{2 a - 1}$ with methyl malonate $\mathbf{5 a}$ under the catalysis of 14 (Table 3). The results collected pointed out the following facts: (i) compounds containing either electron withdrawing or electron donating groups were equally good substrates; (ii) substrates containing aromatic heterocycles were also good substrates giving products $7 \mathbf{e}$ in excellent yields and similar enantiomeric excess (Table 3, entries 7); (iii) alkyl substituted isoxazole 1i reacted equally well giving aliphatic cyclopropane $7 \mathbf{i}$ in comparable yield and ee.

Table 3. Catalytic Asymmetric cyclopropanation of bromostyrylisoxazoles 2a-i with dimethyl malonate ${ }^{[\mathrm{a}]}$.

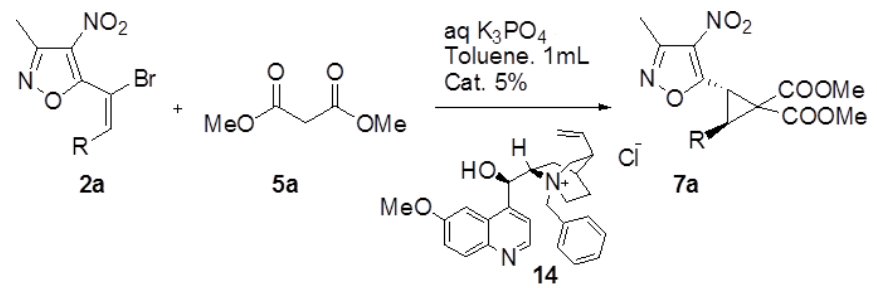

\begin{tabular}{ccccccc}
\hline Entry & $\mathbf{R}$ & Prod. & Time/h & $\begin{array}{c}\text { Yield } \\
{[\mathbf{\%}]^{[\mathbf{b}]}}\end{array}$ & $\begin{array}{c}\boldsymbol{e e} \\
{[\mathbf{\%}]^{[\mathbf{c}]}}\end{array}$ & Isomer \\
\hline 1 & $\mathrm{C}_{6} \mathrm{H}_{5}$ & $\mathbf{7 a}$ & 48 & 97 & 42 & $(-)$ \\
2 & $4-\mathrm{OCH}_{3}-\mathrm{C}_{6} \mathrm{H}_{4}$ & $\mathbf{7 b}$ & 48 & 97 & 45 & $(-)$ \\
3 & $2,3-\mathrm{Cl}_{2}-\mathrm{C}_{6} \mathrm{H}_{3}$ & $\mathbf{7 c}$ & 48 & 95 & 40 & $(-)$ \\
4 & $2-\mathrm{Cl}^{-} \mathrm{C}_{6} \mathrm{H}_{4}$ & $\mathbf{7 d}$ & 48 & 94 & 48 & $(-)$ \\
5 & $2-$ thienyl & $\mathbf{7 e}$ & 48 & 95 & 49 & $(-)$ \\
6 & $2-\mathrm{OMe}-\mathrm{C}_{6} \mathrm{H}_{4}$ & $\mathbf{7 f}$ & 48 & 97 & 56 & $(-)$ \\
7 & $2-\mathrm{Br}_{6} \mathrm{C}_{6} \mathrm{H}_{4}$ & $\mathbf{7 g}$ & 48 & 94 & 54 & $(-)$ \\
8 & $2,4-O M e-\mathrm{C}_{6} \mathrm{H}_{3}$ & $\mathbf{7 h}$ & 48 & 91 & 58 & $(-)$ \\
9 & $n$-heptyl & $\mathbf{7 i}$ & 48 & 96 & 46 & $(-)$ \\
\hline
\end{tabular}

[a] Reaction Conditions: bromostyrylisoxazole 2a-i $(0.1 \mathrm{mmol})$, toluene $(5.0 \mathrm{~mL})$, cat. $14(0.005 \mathrm{mmol})$, dimethyl malonate $5 \mathbf{a}(0.2 \mathrm{mmol}), \mathrm{K}_{3} \mathrm{PO}_{4} 50 \% \mathrm{w} / \mathrm{w}(1 \mathrm{mmol})$. [b] Isolated yields after flash column chromatography. [c] The enantiomeric excess (ee) of the product was determined by chiral stationary phase HPLC. 
We have compared the data collected for the reaction of 1 and 6 [14] with those for the reaction of 2 and malonate $\mathbf{5}$ and explained the observed difference in enantioselectivity as follows (Figure 2). Firstly, the requirement for a free $-\mathrm{OH}$ on the phase transfer catalyst indicates the interaction of this group with one of the two reagents involved, presumably the enolate. It is well known that ${ }^{+} \mathrm{N}-\mathrm{C}_{\alpha} \mathrm{H}$ behaves as strong hydrogen bond donors. [44] Therefore, it is possible that in apolar media such as toluene an interaction could take place between the catalyst ${ }^{+} \mathrm{N}-\mathrm{C}_{\alpha} \mathrm{H}$ and the nitro group of the styrylisoxazole. According to this rationale, the bromine in compounds $\mathbf{2}$ shielded the $\mathrm{NO}_{2}$, limiting its interaction with the PTC as it may occur for compounds 1, hence justifying the lower enantioselectivity observed.

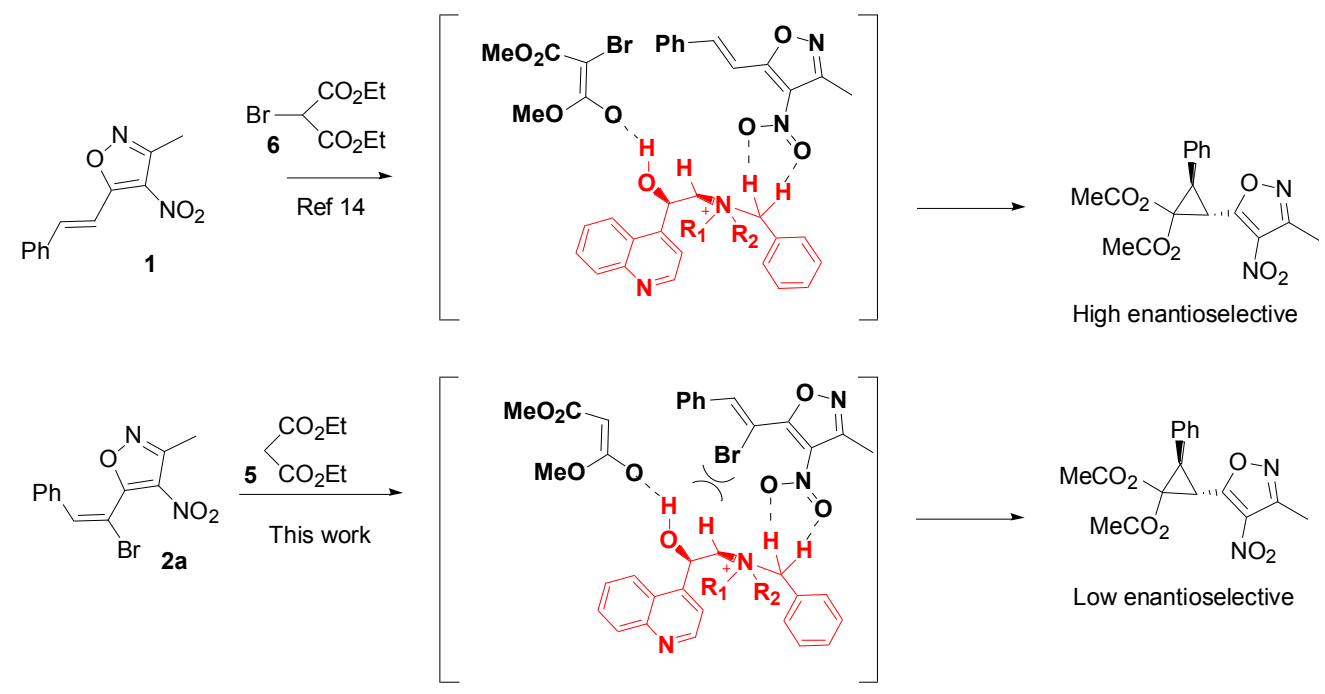

Figure 2. Proposed transition states for the cyclopropanation of $\mathbf{1}$ and $\mathbf{2}$.

\section{Experimental Section}

General procedure for the preparation of compounds $7 \mathbf{a}-\mathbf{i}$ : To a test tube equipped with a magnetic stirring bar were sequentially added the bromostyrylisoxazole $\mathbf{2 a}-\mathbf{1}(0.1 \mathrm{mmol})$, toluene $(1.0 \mathrm{~mL})$, catalyst $14(5 \mathrm{~mol} \%)$ and malonate $5 \mathbf{a}-\mathbf{e}(0.2 \mathrm{mmol})$. The test tube was placed at the stated temperature, then $\mathrm{K}_{3} \mathrm{PO}_{4} 50 \% \mathrm{w} / \mathrm{w}$ was added in one portion $(0.28 \mathrm{~mL}, 1.0 \mathrm{mmol})$. The mixture was then vigorously stirred at the same temperature, with no precautions to exclude moisture or air. After the stated reaction time, the reaction was then quenched with sat. $\mathrm{NH}_{4} \mathrm{Cl}(4 \mathrm{~mL})$ and the product extracted with toluene $(3 \times 1 \mathrm{~mL})$. The combined organic phases were evaporated and the product was then purified by chromatography on silica gel (petroleum ether/EtOAc mixtures).

2-(3-Methyl-4-nitro-isoxazol-5-yl)-3-phenyl-cyclopropane-1,1-dicarboxylic acid dimethyl ester 7a: $R_{\mathrm{f}}=0.1$ (petroleum ether : EtOAc, 95:5); IR (neat)/cm ${ }^{-1}: 2999 \mathrm{w}, 2955 \mathrm{w}, 2875 \mathrm{w}, 1749 \mathrm{~s}, 1550 \mathrm{~s}$; ee determined by CSP-HPLC using a Chiralpak AD column ( $n$-hexane/i-PrOH 90:10, flow rate $1.0 \mathrm{~mL} / \mathrm{min}, t_{1}=14.95 \mathrm{~min}, t_{2}=13.86 \mathrm{~min} .{ }^{1} \mathrm{H}-\mathrm{NMR}\left(400 \mathrm{MHz}, \mathrm{CDCl}_{3}\right): 7.25(\mathrm{Ar}-\mathrm{H}, \mathrm{m}, 5 \mathrm{H})$, 4.19 (Is-CH, d, $1 \mathrm{H}, J=8.4 \mathrm{~Hz}), 3.89(\mathrm{Ar}-\mathrm{CH}, \mathrm{d}, 1 \mathrm{H}, J=8.4 \mathrm{~Hz}), 3.67\left(\mathrm{CO}_{2} \mathrm{CH}_{3}, \mathrm{~s}, 3 \mathrm{H}\right)$, $3.43\left(\mathrm{CO}_{2} \mathrm{CH}_{3}, \mathrm{~s}, 3 \mathrm{H}\right), 2.51$ (Is- $\left.\mathrm{CH}_{3}, \mathrm{~s}, 3 \mathrm{H}\right) ;{ }^{13} \mathrm{C}-\mathrm{NMR}(100.6 \mathrm{MHz}): 167.57,165.25,163.94,154.99$, 131.03, 130.5, 127.56, 127.44, 127.25, 52.48, 52.05, 43.81, 35.28, 25.25, 10.59; HRMS: $m / z$ found $[\mathrm{M}+\mathrm{Na}]^{+} 383.0858, \mathrm{C}_{17} \mathrm{H}_{16} \mathrm{~N}_{2} \mathrm{O}_{7} \mathrm{Na}$ requires 383.0855, $m / z: 383\left(100 \%,[\mathrm{M}+\mathrm{Na}]^{+}\right)$. 
2-(4-Methoxy-phenyl)-3-(3-methyl-4-nitro-isoxazol-5-yl)-cyclopropane-1,1-dicarboxylic

acid dimethyl ester 7b: $R_{\mathrm{f}}=0.1$ (petroleum ether:EtOAc, 75:25); IR (neat) $/ \mathrm{cm}^{-1}: 2999 \mathrm{w}, 2954 \mathrm{w}, 1750 \mathrm{~s}$, $1558 \mathrm{~s}$; ee determined by CSP-HPLC using a Chiralpak AD column ( $n$-hexane/ $i \mathrm{PrOH}$ 80:20, flow rate $1.0 \mathrm{~mL} / \mathrm{min}, t_{1}=18.06 \mathrm{~min}, t_{2}=14.31 \mathrm{~min} .{ }^{1} \mathrm{H}-\mathrm{NMR}\left(400 \mathrm{MHz}, \mathrm{CDCl}_{3}\right): 7.16(\mathrm{Ar}-\mathrm{H}, \mathrm{d}, 2 \mathrm{H}$, $J=8.0 \mathrm{~Hz}), 6.78(\mathrm{Ar}-\mathrm{H}, \mathrm{d}, 2 \mathrm{H}, J=8.0 \mathrm{~Hz}), 4.14(\mathrm{Is}-\mathrm{CH}, \mathrm{d}, 1 \mathrm{H}, J=8.4 \mathrm{~Hz}), 3.83$ (Ar-CH, d, 1H, $J=8.4 \mathrm{~Hz}), 3.72\left(\mathrm{CO}_{2} \mathrm{CH}_{3}, \mathrm{~s}, 3 \mathrm{H}\right), 3.66\left(\mathrm{CO}_{2} \mathrm{CH}_{3}, \mathrm{~s}, 3 \mathrm{H}\right), 3.46\left(\mathrm{Ar}-\mathrm{OCH}_{3}, \mathrm{~s}, 3 \mathrm{H}\right), 2.50\left(\mathrm{Is}-\mathrm{CH}_{3}, \mathrm{~s}\right.$, $3 \mathrm{H}) ;{ }^{13} \mathrm{C}-\mathrm{NMR}$ (100.6 MHz): 168.8, 166.4, 165.1, 159.5, 156.0, 131.0, 129.6, 123.9, 114.0, 55.3, 53.5, 53.1, 44.9, 35.9, 26.4, 11.6; HRMS: $m / z$ found: $[\mathrm{M}+\mathrm{Na}]^{+} 413.0959, \mathrm{C}_{18} \mathrm{H}_{18} \mathrm{~N}_{2} \mathrm{O} 8 \mathrm{Na}$ requires 413.0961, $m / z: 413\left(100 \%,[\mathrm{M}+\mathrm{Na}]^{+}\right)$.

Dimethyl 2-(2,3-dichlorophenyl)-3-(3-methyl-4-nitroisoxazol-5-yl)cyclopropane-1,1-dicarboxylate 7c: $R_{\mathrm{f}}=0.2$ (petroleum ether:EtOAc, 80:20); IR (neat) $/ \mathrm{cm}^{-1}: 3005 \mathrm{w}, 2931 \mathrm{w}, 1740 \mathrm{~s}, 1561 \mathrm{~s}$; ee was determined by CSP-HPLC using a Chiralpak AD column ( $n$-hexane/iPrOH 80:20, flow rate 1.0 $\mathrm{mL} / \mathrm{min}, t_{1}=10.98 \mathrm{~min}, t_{2}=8.98 \mathrm{~min} .{ }^{1} \mathrm{H}-\mathrm{NMR}\left(400 \mathrm{MHz}, \mathrm{CDCl}_{3}\right): 7.46-7.43(\mathrm{~m}, 1 \mathrm{H}), 7.22-7.21(\mathrm{~m}$, 2H), $4.29(\mathrm{~d}, J=8.4 \mathrm{~Hz}, 1 \mathrm{H}), 4.00(\mathrm{~d}, J=8.4 \mathrm{~Hz}, 1 \mathrm{H}), 3.76(\mathrm{~s}, 3 \mathrm{H}), 3.58(\mathrm{~s}, 3 \mathrm{H}), 2.58(\mathrm{~s}, 3 \mathrm{H})$; ${ }^{13} \mathrm{C}-\mathrm{NMR}$ (100.6 MHz): 168.1, 165.8, 165.2, 156.2, 134.1, 133.6, 132.9, 130.6, 128.2, 127.3, 53.7, 53.5, 44.4, 35.8, 29.8, 27.2, 11.7; IR $(\mathrm{NaCl}) / \mathrm{cm}^{-1}: 1754 \mathrm{~s}, 1518 \mathrm{~s} ; \mathrm{HRMS}: \mathrm{m} / z$ found $[\mathrm{M}+\mathrm{H}]^{+}$ $429.0266, \mathrm{C}_{17} \mathrm{H}_{15} \mathrm{~N}_{2} \mathrm{O}_{7} \mathrm{Cl}_{2}$ requires 429.0256 .

Dimethyl 2-(2-chlorophenyl)-3-(3-methyl-4-nitroisoxazol-5-yl)cyclopropane-1,1-dicarboxylate 7d: $R_{\mathrm{f}}=0.3$ (petroleum ether:EtOAc, 80:20); IR (neat) $/ \mathrm{cm}^{-1}: 3016 \mathrm{w}, 2901 \mathrm{w}, 1745 \mathrm{~s}, 1569 \mathrm{~s}$; ee determined by CSP-HPLC using a Chiralpak AD column ( $n$-hexane/iPrOH 90:10, flow rate $1.0 \mathrm{~mL} / \mathrm{min}, t_{1}=14.50 \mathrm{~min}, t_{2}=12.97 \mathrm{~min} .{ }^{1} \mathrm{H}-\mathrm{NMR}\left(400 \mathrm{MHz}, \mathrm{CDCl}_{3}\right): 7.41-7.39(\mathrm{~m}, 1 \mathrm{H})$, $7.31-7.27(\mathrm{~m}, 3 \mathrm{H}), 4.31(\mathrm{~d}, J=8.0 \mathrm{~Hz}, 1 \mathrm{H}), 4.01(\mathrm{~d}, J=8.4,1 \mathrm{H}), 3.76(\mathrm{~s}, 3 \mathrm{H}), 3.55(\mathrm{~s}, 3 \mathrm{H}), 2.58$ (s, $3 \mathrm{H}) ;{ }^{13} \mathrm{C}-\mathrm{NMR}(100.6 \mathrm{MHz}): 168.5,166.0,165.3,156.2,135.8,130.6,129.9,129.8,129.7,126.9$, 53.6, 53.3, 44.4, 35.4, 29.8, 26.9, 11.8; IR $(\mathrm{NaCl}) / \mathrm{cm}^{-1}: 1730 \mathrm{~s}, 1533 \mathrm{~s}$; HRMS: $\mathrm{m} / z$ found $[\mathrm{M}+\mathrm{H}]^{+}$ 395.0636, $\mathrm{C}_{17} \mathrm{H}_{16} \mathrm{~N}_{2} \mathrm{O}_{7} \mathrm{Cl}$ requires 395.0646.

Dimethyl 2-(3-methyl-4-nitroisoxazol-5-yl)-3-(thiophen-2-yl)cyclopropane-1,1-dicarboxylate 7e: $R_{\mathrm{f}}=0.4$ (petroleum ether:EtOAc, 70:30); IR (neat) $/ \mathrm{cm}^{-1}: 2995 \mathrm{w}, 2915 \mathrm{w}, 1749 \mathrm{~s}, 1559 \mathrm{~s}$; ee was determined by CSP-HPLC using a Chiralpak AD column ( $n$-hexane/iPrOH 90:10, flow rate $1.0 \mathrm{~mL} / \mathrm{min}, t_{1}=16.38 \mathrm{~min}, t_{2}=14.81 \mathrm{~min} .{ }^{1} \mathrm{H}-\mathrm{NMR}\left(400 \mathrm{MHz}, \mathrm{CDCl}_{3}\right): 7.24(\mathrm{~d}, J=0.8 \mathrm{~Hz}, 1 \mathrm{H})$, $7.01-7.00(\mathrm{~m}, 1 \mathrm{H}), 6.97-6.95(\mathrm{~m}, 1 \mathrm{H}), 4.18(\mathrm{~d}, J=8.0 \mathrm{~Hz}, 1 \mathrm{H}), 3.99(\mathrm{~d}, J=8.0 \mathrm{~Hz}, 1 \mathrm{H}), 3.73(\mathrm{~s}, 3 \mathrm{H})$, 3.61 (s, 3H), 2.57 (s, 3H); ${ }^{13} \mathrm{C}-\mathrm{NMR}$ (100.6 MHz): 168.0, 166.1, 164.8, 156.1, 134.8, 127.5, 127.1, 126.3, 53.7, 53.4, 45.3, 31.3, 29.8, 28.2, 11.7; IR $(\mathrm{NaCl}) / \mathrm{cm}^{-1}: 1734 \mathrm{~s}, 1549 \mathrm{~s} ; \mathrm{HRMS}: m / z$ found $[\mathrm{M}+\mathrm{H}]^{+} 367.0582, \mathrm{C}_{15} \mathrm{H}_{15} \mathrm{~N}_{2} \mathrm{O}_{7} \mathrm{~S}$ requires 367.0600 .

Dimethyl 2-(2-methoxyphenyl)-3-(3-methyl-4-nitroisoxazol-5-yl)cyclopropane-1,1-dicarboxylate 7f: $R_{\mathrm{f}}=0.2$ (petroleum ether:EtOAc, 85:15); IR (neat) $/ \mathrm{cm}^{-1}: 2980 \mathrm{w}, 2815 \mathrm{w}, 1745 \mathrm{~s}, 1569 \mathrm{~s}$; ee was determined by CSP-HPLC using a Chiralcel OD column ( $n$-hexane/i-PrOH 95:5, flow rate $0.50 \mathrm{~mL} / \mathrm{min}, t_{1}=39.46 \mathrm{~min}, t_{2}=29.21 \mathrm{~min} .{ }^{1} \mathrm{H}-\mathrm{NMR}\left(400 \mathrm{MHz}, \mathrm{CDCl}_{3}\right): 7.31-7.27(\mathrm{~m}, 1 \mathrm{H})$, $7.18-7.16(\mathrm{~m}, 1 \mathrm{H}), 6.94-6.90(\mathrm{~m}, 1 \mathrm{H}), 6.87(\mathrm{~d}, J=8.4 \mathrm{~Hz}, 1 \mathrm{H}), 4.23(\mathrm{~d}, J=8.4 \mathrm{~Hz}, 1 \mathrm{H}), 3.94(\mathrm{~d}$, $J=8.4 \mathrm{~Hz}, 1 \mathrm{H}), 3.83(\mathrm{~s}, 3 \mathrm{H}), 3.73(\mathrm{~s}, 3 \mathrm{H}), 3.51$ (s, 3H), $2.58(\mathrm{~s}, 3 \mathrm{H}) ;{ }^{13} \mathrm{C}-\mathrm{NMR}(100.6 \mathrm{MHz}): 169.3$, 166.6, 165.6, 158.7, 156.1, 129.7, 129.2, 129.0, 128.4, 120.9, 120.4, 110.5, 55.7, 53.5, 53.0, 44.4, 32.9, 
29.8, 26.7, 11.8; IR (NaCl)/cm ${ }^{-1}: 1734$ s, 1518 s; HRMS: $m / z$ found $[\mathrm{M}-\mathrm{H}]^{-} 389.0966, \mathrm{C}_{18} \mathrm{H}_{17} \mathrm{~N}_{2} \mathrm{O}_{8}$ requires 389.0985 .

Dimethyl 2-(2-bromophenyl)-3-(3-methyl-4-nitroisoxazol-5-yl)cyclopropane-1,1-dicarboxylate 7g: $R_{\mathrm{f}}=0.3$ (petroleum ether:EtOAc, 80:20); IR (neat) $/ \mathrm{cm}^{-1}: 2988 \mathrm{w}, 2810 \mathrm{w}, 1750 \mathrm{~s}, 1561 \mathrm{~s}$; ee determined by CSP-HPLC using a Chiralpak AD column ( $n$-hexane/i-PrOH 90:10, flow rate $1.0 \mathrm{~mL} / \mathrm{min}, t_{1}=16.78 \mathrm{~min}, t_{2}=15.22 \mathrm{~min} .{ }^{1} \mathrm{H}-\mathrm{NMR}\left(400 \mathrm{MHz}, \mathrm{CDCl}_{3}\right): 7.60-7.58(\mathrm{~m}, 1 \mathrm{H})$, $7.33-7.26(\mathrm{~m}, 2 \mathrm{H}), 7.23-7.18(\mathrm{~m}, 1 \mathrm{H}), 4.31(\mathrm{~d}, J=8.4 \mathrm{~Hz}, 1 \mathrm{H}), 3.99$ (d, $J=8.6 \mathrm{~Hz}, 1 \mathrm{H}), 3.76(\mathrm{~s}, 3 \mathrm{H})$, 3.55 (s, 3H), 2.58 (s, 3H); ${ }^{13} \mathrm{C}-\mathrm{NMR}$ (100.6 MHz): 168.4, 165.9, 165.2, 156.2, 133.0, 132.3, 130.0, 130.0, 127.5, 125.8, 53.6, 53.3, 44.6, 37.6, 27.2, 11.8; IR $(\mathrm{NaCl}) / \mathrm{cm}^{-1}: 1738 \mathrm{~s}, 1522 \mathrm{~s}$; HRMS: $\mathrm{m} / \mathrm{z}$ found $[\mathrm{M}-\mathrm{H}]^{-}$436.9993, $\mathrm{C}_{17} \mathrm{H}_{14} \mathrm{~N}_{2} \mathrm{O}_{7} \mathrm{Br}$ requires 436.9984 .

Dimethyl 2-(2,4-dimethoxyphenyl)-3-(3-methyl-4-nitroisoxazol-5-yl)cyclopropane-1,1-dicarboxylate $7 \mathbf{h}: R_{\mathrm{f}}=0.3$ (petroleum ether:EtOAc, 70:30); IR (neat) $/ \mathrm{cm}^{-1}: 2980 \mathrm{w}, 2815 \mathrm{w}, 1745 \mathrm{~s}, 1569 \mathrm{~s}$; ee was determined by CSP-HPLC using a Chiralcel OD column ( $n$-hexane/i-PrOH 80:20, flow rate 0.75 $\mathrm{mL} / \mathrm{min}, t_{1}=23.57 \mathrm{~min}, t_{2}=20.01 \mathrm{~min} .{ }^{1} \mathrm{H}-\mathrm{NMR}$ (400 $\left.\mathrm{MHz}, \mathrm{CDCl}_{3}\right): 7.08-7.05(\mathrm{~m}, 1 \mathrm{H}), 6.44-6.42$ $(\mathrm{m}, 2 \mathrm{H}), 4.18(\mathrm{~d}, J=8.4 \mathrm{~Hz}, 1 \mathrm{H}), 3.87(\mathrm{~d}, J=8.4 \mathrm{~Hz}, 1 \mathrm{H}), 3.80(\mathrm{~s}, 3 \mathrm{H}), 3.79$ (s, 3H), $3.72(\mathrm{~s}, 3 \mathrm{H})$, 3.54 (s, 3H), 2.57 (s, 3H); ${ }^{13} \mathrm{C}-\mathrm{NMR}$ (100.6 MHz): 169.4, 166.7, 165.6, 161.1, 159.7, 156.1, 129.5, 113.2, 104.0, 98.6, 55.7, 55.5, 53.4, 53.1, 44.4, 32.7, 29.8, 26.7, 11.8; IR (NaCl)/cm ${ }^{-1}: 1734 \mathrm{~s}, 1510 \mathrm{~s}$; HRMS: $m / z$ found $[\mathrm{M}-\mathrm{H}]^{-} 419.1109, \mathrm{C}_{19} \mathrm{H}_{19} \mathrm{~N}_{2} \mathrm{O}_{9}$ requires 419.1091 .

Dimethyl 2-hexyl-3-(3-methyl-4-nitroisoxazol-5-yl)cyclopropane-1,1-dicarboxylate $\quad 7 \mathrm{i}: \quad R_{\mathrm{f}}=0.4$ (petroleum ether:EtOAc, 60:40); IR (neat) $/ \mathrm{cm}^{-1}: 2995$ w, 2845 w, 1751s, 1560s; ee was determined by CSP-HPLC using a Chiralpak AD column ( $n$-hexane $/ i-\mathrm{PrOH}$ 95:5, flow rate $1.0 \mathrm{~mL} / \mathrm{min}, t_{1}=7.49 \mathrm{~min}$, $t_{2}=6.26 \mathrm{~min} .{ }^{1} \mathrm{H}-\mathrm{NMR}\left(400 \mathrm{MHz}, \mathrm{CDCl}_{3}\right): 3.83-3.82(\mathrm{~m}, 4 \mathrm{H}), 3.65(\mathrm{~s}, 3 \mathrm{H}), 3.55(\mathrm{~d}, J=7.6 \mathrm{~Hz}, 1 \mathrm{H})$, $2.53(\mathrm{~s}, 3 \mathrm{H}), 1.64-1.58(\mathrm{~m}, 2 \mathrm{H}), 1.49-1.40(\mathrm{~m}, 2 \mathrm{H}), 1.34-1.24(\mathrm{~m}, 6 \mathrm{H}), 0.89-0.85 \mathrm{~m}(\mathrm{~m}, 3 \mathrm{H})$; ${ }^{13} \mathrm{C}-\mathrm{NMR}$ (100.6 MHz): 169.4, 166.8, 166.5, 165.1, 156.0, 54.1, 53.4, 43.2, 41.7, 33.1, 31.7, 28.8, 28.1, 27.2, 22.6, 14.1, 11.7; IR $(\mathrm{NaCl}) / \mathrm{cm}^{-1}: 1750 \mathrm{~s}, 1526 \mathrm{~s}$; HRMS: $\mathrm{m} / z$ found $[\mathrm{M}-\mathrm{H}]^{-} 367.1512$, $\mathrm{C}_{17} \mathrm{H}_{23} \mathrm{~N}_{2} \mathrm{O}_{7}$ requires 367.1505 .

\section{Conclusions}

In conclusion, we have reported a new method for the preparation of heavily substituted cyclopropane esters $\mathbf{7 a}-\mathbf{i}$. These compounds were prepared in high yields, as a single diastereoisomer and in average (up to 58\%) enantioselectivity. We interpreted the low enantioselectivity observed as an indirect proof for bi-functional mode of action of Cinchona phase transfer catalysts.

\section{Acknowledgments}

We acknowledge Science Foundation Ireland (SFI) for a grant to LP, the Irish Research Council for Science and Engineering (IRCSET) for a grant to MC and Fondazione con il Sud for a grant to MM. 


\section{Author Contributions}

M.F.A.A. conceived and designed the experiments; L.P. M.C. and M.M. performed the experiments; M.F.A.A. analyzed the data; W.W. M.F.A.A. M.M. and M.C. wrote the paper.

\section{References}

1. Adamo, M.F.A.; Duffy, E.F.; Konda, V.R.; Murphy, F. An improved synthesis of 3-methyl-4nitro-5-heteroarylethenylisoxazoles. Heterocycles 2007, 71, 1173-1181.

2. Adamo, M.F.A.; Duffy, E.F. Multicomponent synthesis of 3-heteroarylpropionic acids. Org. Lett. 2006, 8, 5157-5159.

3. Adamo, M.F.A.; Suresh, S.; Piras, L. Reaction of 5-(1-bromo-2-arylvinyl)-3-methyl-4nitroisoxazoles and 1,3-dicarbonyl compounds. Tetrahedron 2009, 65, 5402-5408.

4. Adamo, M.F.A.; Chimichi, S.; De Sio, F.; Donati, D.; Sarti-Fantoni, P. The reactivity of 3-methyl-4nitro-5-styrylisoxazole with some bis-enolisable ketones. Tetrahedron Lett. 2002, 43, 4157-4160.

5. Adamo, M.F.A.; Donati, D.; Duffy, E.F.; Sarti-Fantoni, P. Multicomponent synthesis of spiroisoxazolines. J. Org. Chem. 2005, 70, 8395.

6. Adamo, M.F.A.; Duffy, E.F.; Donati, D.; Sarti-Fantoni, P. Modular synthesis of isoxazolepyridones and pyrazolepyridones. Tetrahedron 2007, 63, 2047-2052.

7. Adamo, M.F.A.; Konda, V.R. Multicomponent synthesis of 3-indolepropionic acids. Org. Lett. 2007, 9, 303-305.

8. Fini, F.; Nagabelli, M.; Adamo, M.F.A. Development of a Mild Procedure for the Addition of Bisulfite to Electrophilic Olefins. Adv. Synth. Catal. 2010, 352, 3163-3168.

9. Baschieri, A.; Bernardi, L.; Ricci, A.; Suresh, S.; Adamo, M.F.A. Catalytic Asymmetric Conjugate Addition of Nitroalkanes to 4-Nitro-5-styrylisoxazoles. Angew. Chem. Int. Ed. 2009, 48, 9342-9345.

10. Wells, R; Moccia, M.; Adamo, M.F.A. The preparation of 3-methyl-4-nitro-5-(2alkylethenyl)isoxazoles. Tetrahedron Lett. 2014, 55, 803-805.

11. Chimichi, S.; De Sio, F.; Donati, D.; Fina, G.; Pepino R.; Sarti-Fantoni, P. The preparation of coumaric acids via styrylisoxazoles. Heterocycles 1983, 20, 263-267.

12. Kawai, H.; Tachi, K.; Tokunaga, E.; Shiro, M.; Shibata, N. Trifluoromethylation of Aromatic Isoxazoles: Regio- and Diastereoselective route to 5-Trifluoromethyl-2-isoxazolines. Angew. Chem. Int. Ed. 2011, 50, 7803-7806.

13. Kawai, H.; Sugita, Y.; Tokunaga, E.; Sato, H.; Shiro, M.; Shibata, N. Diastereoselective Additive Trifluoromethylation/Halogenation of Isoxazole Triflones: Synthesis of All-CarbonFunctionalized Trifluoromethyl Isoxazoline Triflones. Chemistry Open. 2014; 3, 14-18.

14. Wang, X.; Tokunaga, E.; Shibata, N. Direct nucleophilic difluoromethylation of aromatic isoxazoles activated by electron-withdrawing groups using (difluoromethyl)trimethylsilane. ScienceOpen Res. 2015; doi:10.14293/S2199-1006.1.SOR-CHEM.AD1QVW.v1.

15. Pietruszka, J. Synthesis and Properties of Oligocyclopropyl-Containing Natural Products and Model Compounds. Chem. Rev. 2003, 103, 1051-1070. 
16. Donaldson, W.A. Synthesis of cyclopropane containing natural products. Tetrahedron 2001, 57, 8589-8627.

17. Lebel, H.; Marcoux, J.F.; Molinaro, C.; Charet, A.B. Stereoselective Cyclopropanation Reactions. Chem. Rev. 2003, 103, 977-1050.

18. Aggarwal, V.K.; Smith, H.W.; Jones, R.V. H.; Fieldhouse, R. Catalytic Asymmetric Cyclopropanation of Electron Deficient Alkenes Mediated By Chiral Sulfides. Chem. Commun. 1998, 1785-1786.

19. Aggarwal, V.K.; Alonso, E.; Fang, G.; Ferrara, M.; Hynd, G.; Porcelloni, M. Application of Chiral Sulfides to Catalytic Asymmetric Aziridination and Cyclopropanation with In Situ Generation of the Diazo Compound. Angew. Chem. Int. Ed. 2001, 40, 1433-1436.

20. Zheng, J.C.; Liao, W.W.; Tang, Y.; Sun, X.L.; Dai, L.-X. The Michael Addition-Elimination of Ylides to $\alpha, \beta$-Unsaturated Imines. Highly Stereoselective Synthesis of Vinylcyclopropanecarbaldehydes and Vinylcyclopropylaziridines. J. Am. Chem. Soc. 2005, 127, 12222-12223.

21. Deng, X.; Cai, P.; Ye, S.; Sun, X.; Liao, W.; Li, K.; Tang, Y.; Wu, Y.D.; Dai, L.X. Enantioselective Synthesis of Vinylcyclopropanes and Vinylepoxides Mediated by CamphorDerived Sulfur Ylides: Rationale of Enantioselectivity, Scope, and Limitation. J. Am. Chem. Soc. 2006, 128, 9730-9740.

22. Aggarwal, V.K.; Grange, E. Asymmetric Sulfonium Ylide Mediated Cyclopropanation: Stereocontrolled Synthesis of (+)-LY354740. Chem. Eur. J. 2006, 12, 568.

23. Special Issue on "Organocatalysis"; List, B., Ed.; ACS: Washington, D.C, USA, 2007; pp. 5413-5883.

24. Houk, K.N.; List, B. Asymmetric Organocatalysis. Acc. Chem. Res. 2004, 37, 487.

25. Bremeyer, N.; Smith, S.C.; Ley, S.V.; Gaunt, M.J. An Intramolecular Organocatalytic Cyclopropanation Reaction. Angew. Chem. Int. Ed. 2004, 43, 2681-2684.

26. Papageorgiou, C.D.; Cubillo de Dios, M.A.; Ley, S.V.; Gaunt, M.J. Enantioselective Organocatalytic Cyclopropanation via Ammonium Ylides. Angew. Chem. Int. Ed. 2004 43, 4641-4644.

27. Johansson, C.C.C.; Bremeyer, N.; Ley, S.V.; Owen, D.R.; Smith, S.C.; Gaunt, M.J. Enantioselective Catalytic Intramolecular Cyclopropanation using Modified Cinchona Alkaloid Organocatalysts. Angew. Chem. Int. Ed. 2006, 45, 6024-6028.

28. Belyk, K.M., Xiang, B.; Bulger, P.G.; Leonard, W.R., Balsells, J.; Yin, J.; Chen, C. Enantioselective Synthesis of $(1 R, 2 S)$-1-Amino-2-vinylcyclopropanecarboxylic Acid Ethyl Ester (Vinyl-ACCA-OEt) by Asymmetric Phase-Transfer Catalyzed Cyclopropanation of $(E)-N$ Phenylmethyleneglycine Ethyl Ester. Org. Proc. Res. Develop. 2010, 14, 692-700.

29. Herchl, R.; Waser, M. Asymmetric cyclopropanation of chalcones using chiral phase-transfer catalysts. Tetrahedron Lett. 2013, 54, 2472-2475.

30. Kunz, R.K.; MacMillan, D.W.C. Enantioselective Organocatalytic Cyclopropanations. The Identification of a New Class of Iminium Catalyst Based upon Directed Electrostatic Activation. J. Am. Chem. Soc. 2005, 127, 3240-3241.

31. McCooey, S.H.; McCabe, T.; Connon, S.J. Stereoselective Synthesis of Highly Functionalized Nitrocyclopropanes via Organocatalyic Conjugate Addition to Nitroalkenes. J. Org. Chem. 2006, $71,7494-7497$. 
32. Hansen, H.M.; Longbottom, D.A.; Ley, S.V. A new asymmetric organocatalytic nitrocyclopropanation reaction. Chem. Commun. 2006, 4838-4840.

33. Xie, H.; Zu, L.; Li, H.; Wang, J.; Wang, W.J. Organocatalytic Enantioselective Cascade MichaelAlkylation Reactions: Synthesis of Chiral Cyclopropanes and Investigation of Unexpected Organocatalyzed Stereoselective Ring Opening of Cyclopropanes. J. Am. Chem. Soc. 2007, 129, 10886-10894.

34. Rios, R.; Sunden, H.; Vesely, J.; Zhao, G.L.; Dziedzic, P.; Cordova, A. A Simple Organocatalytic Enantioselective Cyclopropanation of $\alpha, \beta$-Unsaturated Aldehydes. Adv. Synth. Catal. 2007, 349, 1028-1032.

35. Hartikka, A.; Slosarczyk, A.T.; Arvidsson, P.I. Application of novel sulfonamides in enantioselective organocatalyzed cyclopropanation. Tetrahedron 2007, 18, 1403-1409.

36. Wascholowski, V.; Hansen, H.M.; Longbottom, D.A.; Ley, S.V. A general organocatalytic enantioselective nitrocyclopropanation reaction. Synthesis 2008, 1269-1275.

37. Ibrahem, I.; Zhao, G.L.; Rios, R.; Vesely, J.; Sunden, H.; Dziedzic, P.; Cordova, A. One-Pot Organocatalytic Domino Michael/ $\alpha$-Alkylation Reactions: Direct Catalytic Enantioselective Cyclopropanation and Cyclopentanation Reactions. Chem. Eur. J. 2008, 14, 7867-7879.

38. Lv, J.; Zhang, J.; Lin, Z.; Wang, Y. Enantioselective Synthesis of Functionalized Nitrocyclopropanes by Organocatalytic Conjugate Addition of Bromonitroalkanes to $\alpha, \beta-$ Unsaturated Enones. Chem. Eur. J. 2009, 15, 972-979.

39. Xuan, Y.; Nie, S.; Dong, L.; Zhang, J.; Yan, M. Highly Enantioselective Synthesis of Nitrocyclopropanes via Organocatalytic Conjugate Addition of Bromomalonate to $\alpha, \beta$ Unsaturated Nitroalkenes. Org. Lett. 2009, 11, 1583-1586.

40. Marini, F.; Sternativo, S.; Del Verme, F.; Testaferri, L.; Tiecco, M.A. New Stereoselective Synthesis of Cyclopropanes Containing Quaternary Stereocentres via Organocatalytic Michael Addition to Vinyl Selenones. Adv. Synth. Catal. 2009, 351, 1801-1806.

41. Ooi, T.; Maruoka, K. Recent advances in asymmetric phase-transfer catalysis. Angew.Chem Int. Ed. Engl. 2007, 46, 4222-4266.

42. Arai, S.; Nakayama, K.; Ishida, T.; Shioiri, T. Asymmetric cyclopropanation reaction under phase-transfer catalyzed conditions. Tetrahedron: Lett. 1999, 40, 4215-4218.

43. Del Fiandra, C.; Piras, L.; Fini, F.; Disetti, P.; Moccia, M.; Adamo, M.F.A. Phase transfer catalyzed enantioselective cyclopropanation of 4-nitro-5-styrylisoxazoles. Chem Commun. 2012, $48,3863-3865$.

44. Gomez, B.E.; Linden, A.; López, R.; Mendiola, I.M.; Oiarbide, M.; Palomo, C. Asymmetric Aza-Henry Reaction Under Phase Transfer Catalysis: An Experimental and Theoretical Study. J. Am. Chem. Soc. 2008, 130, 7955-7966.

(C) 2015 by the authors; licensee MDPI, Basel, Switzerland. This article is an open access article distributed under the terms and conditions of the Creative Commons Attribution license (http://creativecommons.org/licenses/by/4.0/). 